\title{
Universiteit
}

Leiden

The Netherlands

\section{Enhanced-fluorescence correlation spectroscopy at micromolar dye concentration around a single gold nanorod}

\author{
Khatua, S.K.; Yuan, H.; Orrit, M.A.G.J.
}

\section{Citation}

Khatua, S. K., Yuan, H., \& Orrit, M. A. G. J. (2015). Enhanced-fluorescence correlation spectroscopy at micromolar dye concentration around a single gold nanorod. Physical Chemistry Chemical Physics, 17(33), 21127-21132. doi:10.1039/C4CP03057E

Version: Publisher's Version

License: $\quad$ Licensed under Article 25fa Copyright Act/Law (Amendment Taverne)

Downloaded from: https://hdl.handle.net/1887/3198536

Note: To cite this publication please use the final published version (if applicable). 


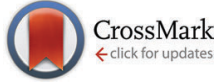

Cite this: Phys. Chem. Chem. Phys., 2015, 17, 21127

Received 11th July 2014 Accepted 29th August 2014

DOI: $10.1039 / c 4 c p 03057 e$

www.rsc.org/pccp

\title{
Enhanced-fluorescence correlation spectroscopy at micro-molar dye concentration around a single gold nanorod $\dagger$
}

\author{
Saumyakanti Khatua, Haifeng Yuan‡ and Michel Orrit*
}

\begin{abstract}
Fluorescence correlation spectroscopy (FCS) is a standard tool for studying diffusion of molecules in solution, but is limited to low analyte concentrations, in the range between $10 \mathrm{pM}$ and $1 \mathrm{nM}$. Such concentration limitations can be overcome by using a plasmonic nanoantenna which confines the electric field of excitation light into a tiny volume near its surface and thereby reduces the effective excitation volume by several orders of magnitude. Here we demonstrate successful FCS measurements on a $1 \mu \mathrm{M}$ solution of crystal violet (CV) dye in glycerol using a gold nanorod antenna. Our correlation analysis yields two components: (i) a slow component with correlation time of about $100 \mathrm{~ms}$, which is attributed to sticking and bleaching of the dye, and (ii) a fast component of about $1 \mathrm{~ms}$, which could arise from dye diffusion through the near-field of the nanorod and/or from blinking due to intersystem crossing or photochemistry.
\end{abstract}

\section{Introduction}

Fluorescence correlation spectroscopy (FCS) is based on statistical analysis of fluctuations in the fluorescence signal. ${ }^{1}$ In a standard FCS experiment, a laser beam is tightly focused into a very dilute solution of fluorophores. While passing through the diffraction-limited focal volume, a fluorophore gives a burst of fluorescence. The autocorrelation of a fluorescence time trace yields the bursts' duration, which provides information on any event causing fluctuations of the fluorescence signal at time scales shorter than the fluorophores' diffusion time through the focal volume. Some examples of such events are conformational changes, bleaching, dwell times in the triplet state, and molecular diffusion. FCS has been used extensively to measure these dynamics in many complex systems in biology and chemistry. ${ }^{1-5}$ FCS, however, requires a very low concentration of fluorophores, typically in the range of tens of pM to a few nM, so that the contrast of the fluorescence bursts dominates the experimental noise. ${ }^{6}$ This requirement restricts the applicability of FCS in many cases, particularly in biology where many reactions occur at micromolar concentrations. ${ }^{7-9}$

FCS at high fluorophore concentrations can be performed by reducing the observation volume well beyond what is achieved by conventional diffraction-limited optics. The most prominent

MoNOS, Huygens-Kamerlingh Onnes Laboratory, Leiden University, 2300 RA Leiden, The Netherlands. E-mail: orrit@physics.leidenuniv.nl

$\dagger$ Electronic supplementary information (ESI) available. See DOI: 10.1039/ c4cp03057e

\$ Present address: Department of Chemistry, KU Leuven, Celestijnenlaan 200F, B-3001 Leuven, Belgium. example of such an approach was from the group of Webb using zero-mode-waveguides which consist of sub-wavelength holes in aluminum films. ${ }^{10}$ These waveguides do not have any propagating mode. The rapid decay of the incident field provides an effective observation volume in the order of a few zeptoliters $\left(1 \mathrm{zL}=10^{-21} \mathrm{~L}\right)$ only. This focal volume is approximately six orders of magnitude smaller than the typical focal volume ( $\sim 1$ femtoliter) achieved by a conventional microscope. Using these waveguides, FCS experiments were successfully performed in solutions with fluorophore concentrations as high as $200 \mu \mathrm{M} .^{10}$

Reduction of the observation volume can also be achieved with plasmonic nanoantennas which confine the incident optical field into a tiny volume of $\sim(10 \mathrm{~nm})^{3}=1 \mathrm{zL}$ near the metal surface. ${ }^{11-13}$ One advantage of these plasmonic nanoantennas over the zero-mode-waveguides is the strong electromagnetic field enhancement associated with the surface plasmon resonances (SPR) of the nanoantenna. Such a concentrated field can strongly enhance the fluorescence of a single molecule. For example, a bowtie or a gold nanorod antenna can enhance single-molecule fluorescence by more than 1000-fold. ${ }^{14,15}$ This strong enhancement of fluorescence also brings on the prospect of generalizing FCS to many weakly emitting species. ${ }^{16}$

Several groups have demonstrated FCS experiments at high fluorophore concentrations by using various plasmonic nanoantennas. Prominent examples include bowtie nanoantennas by Moerner's group and corrugated nanoantennas by the Wenger group. ${ }^{16,17}$ Very recently, Wenger's group have introduced an 'antenna-in-box' platform consisting of a plasmonic nanoantenna and an aperture to further reduce the background 
signal from the unenhanced molecules. ${ }^{8}$ Using these antennas, they reported FCS experiments at analyte concentrations of up to $10 \mu \mathrm{M}$. The above-mentioned plasmonic nanoantennas are fabricated by various lithography methods. Lithography techniques allow one to make nanostructures of various shapes over a large area. The cost of production, however, is usually high because: (1) lithography requires state-of-the-art equipment and cleanroom facilities and (2) metals are often deposited (e.g. in e-beam lithography) through evaporation and hence the yield is poor. Moreover, nanostructures made by evaporation of metals are known to have broad and weaker plasmon resonances because: (a) they are polycrystalline and (b) the metal adhesion layers (Ti or $\mathrm{Cr}$ ) dampen the plasmon resonance. ${ }^{18}$

Alternative bottom-up methods involving wet-chemical synthesis have also been developed to fabricate plasmonic nanostructures. Wet-chemical synthesis has many advantages: (a) it is cheaper and easier than lithography, (b) it can produce single-crystalline particles, and (c) assemblies of nanostructures with very small gaps of a few nanometers can be prepared. This is important to create plasmonic hotspots at the gaps between two closely spaced nanoparticles. These plasmonic hot spots can create strong field enhancements which can be several orders of magnitude larger than those of individual particles. For example, a hot spot between two gold nanospheres can generate a field intensity enhancement larger than 1000 compared to a moderate enhancement of $\sim 5$ by individual nanospheres. ${ }^{9,19}$ With such plasmonic hot-spots in dimers of gold nanospheres or in clusters of silver nanospheres, fluorescence enhancement of over hundred-fold can be obtained. ${ }^{20,21}$ Making such assemblies of nanoparticles and controlling the gap between them, however, is still very difficult and often requires additional templates. For example, DNA molecules were used as templates for the preparation of dimers of nanoparticles by the groups of Tinnefeld and Bidault. ${ }^{20,22}$ Gold nanorods can be a simple alternative to templated assemblies of nanoparticles. These nanoparticles can be synthesized reproducibly in large quantities through simple chemical methods. $^{23-25}$ They create moderately strong field enhancements at the tips when excited at their surface plasmon resonance. ${ }^{12,26,27}$ Such a strong field enhancement was shown to enhance the fluorescence of a weak emitter by 1000 -fold. ${ }^{15}$ Moreover, facile surface functionalization allows one to disperse the nanorods in a broad range of solvents which brings along the prospect of their application in complex systems, e.g., live cells. $^{23,28}$

In this communication, we demonstrate the first FCS experiment on a weakly emitting fluorophore at micromolar concentration using a single gold nanorod. Our experiment consists of gold nanorods immobilized on a polymer surface (PMMA) and covered with a $1 \mu \mathrm{M}$ solution of a fluorophore with a weak quantum yield (crystal violet, $\mathrm{CV}$ ) in glycerol. The fluorescence time traces recorded on single gold nanorods show fluorescence bursts from enhanced CV molecules passing through the near-field. The autocorrelation analysis of such fluorescence time traces reveals two components: (a) a fast component (correlation time of $\sim 1 \mathrm{~ms}$ ) corresponding to the free diffusion of CV molecules through the near-field, and/ or to triplet blinking and (b) a slow component (correlation time $\sim 100 \mathrm{~ms}$ ) which is assigned to sticking and bleaching of dyes at the glass-glycerol interface near the gold nanorod's tips.

\section{Results and discussion}

Single-particle spectroscopy was performed on a home-built confocal sample-scanning microscope as shown in Fig. 1a. Gold nanorods were synthesized chemically and were isolated on a PMMA coated glass substrate through spin coating. The nanorods were then
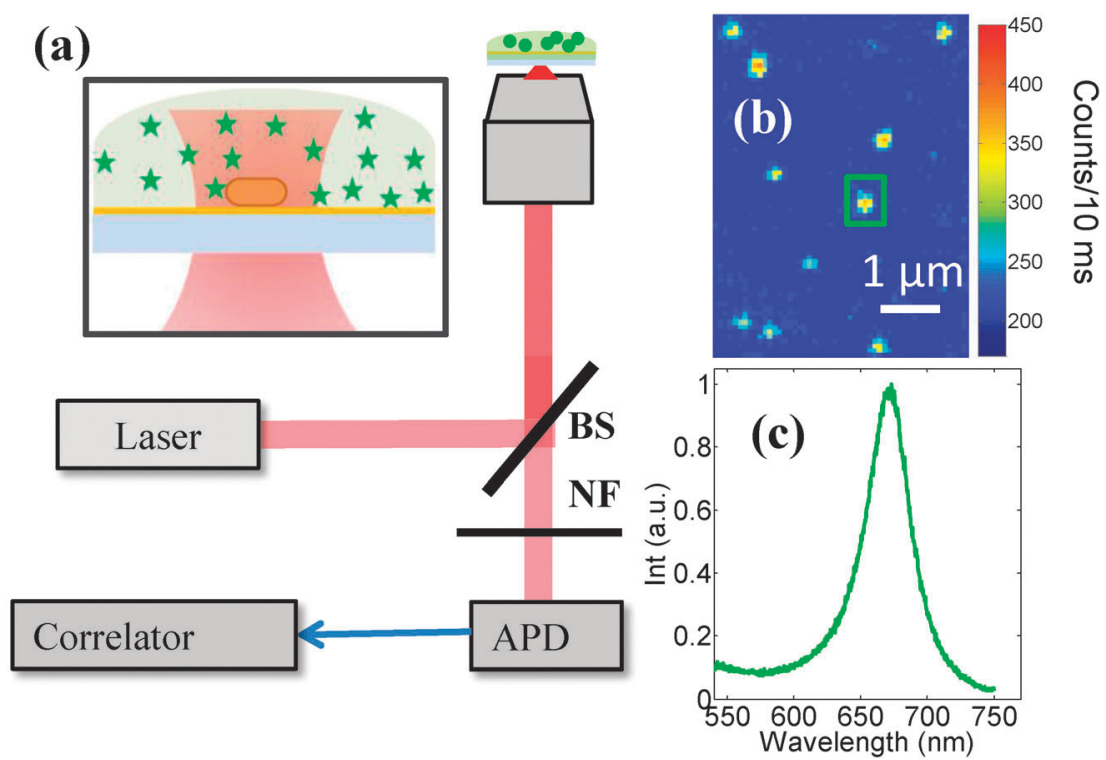

Fig. 1 (a) A simple schematics of the experimental setup. NF and BS stand for notch filter and beam splitter respectively. (b) A typical one-photonluminescence image of single gold nanorods isolated on a glass coverslip and covered with $1 \mu \mathrm{M} \mathrm{CV}$ in glycerol. A circularly polarized $532 \mathrm{~nm}$ laser was used as excitation source. (c) One-photon-luminescence spectrum (green line) of the gold nanorod shown in the green box in (c), in the dye solution. 
covered by a solution of a weakly emitting fluorophore (crystal violet, $1 \mu \mathrm{M}$, fluorescence yield $2 \%$ ) in glycerol. Fig. $1 \mathrm{~b}$ shows a typical one-photon-luminescence image of individual gold nanorods covered by the dye solution. The diffraction-limited bright spots in this image originate from the luminescence of gold nanorods and from enhanced dye fluorescence. We confirmed that these spots stem from single gold nanorods by recording their luminescence spectrum which presented narrow Lorentzian lineshapes. ${ }^{29}$ Fig. 1c shows a typical one-photon-excited luminescence spectrum of a single gold nanorod. Gold nanospheres or aggregates of nanorods can be easily recognized from their spectra, which are either blue shifted (SPR $550 \mathrm{~nm}$ for nanospheres) or have a broad non-Lorentzian line-shape (aggregates of nanoparticles) and were not included in the forthcoming discussion. ${ }^{30}$

To perform correlation spectroscopy, we measured fluorescence time traces of individual gold nanorods. It is important to note that we used $633 \mathrm{~nm}$ excitation wavelength to record the fluorescence time traces. This wavelength selection is based on our previous study where we showed that a maximum fluorescence enhancement is achieved when the excitation energy matches the SPR of a gold nanorod. ${ }^{27}$ Fig. 2a (red) shows a typical time trace recorded on a gold nanorod in a $1 \mu \mathrm{M} \mathrm{CV}$ solution in glycerol at $633 \mathrm{~nm}$ excitation. The enhancement of single-molecule fluorescence is clearly visible as the fluorescence bursts in the time trace recorded on a gold nanorod (red curve in Fig. 2a). Note that no such bursts of fluorescence are seen when a time trace is recorded under same experimental condition but on a place where no nanorod is present (green curve in Fig. 2a). These observations are consistent with our

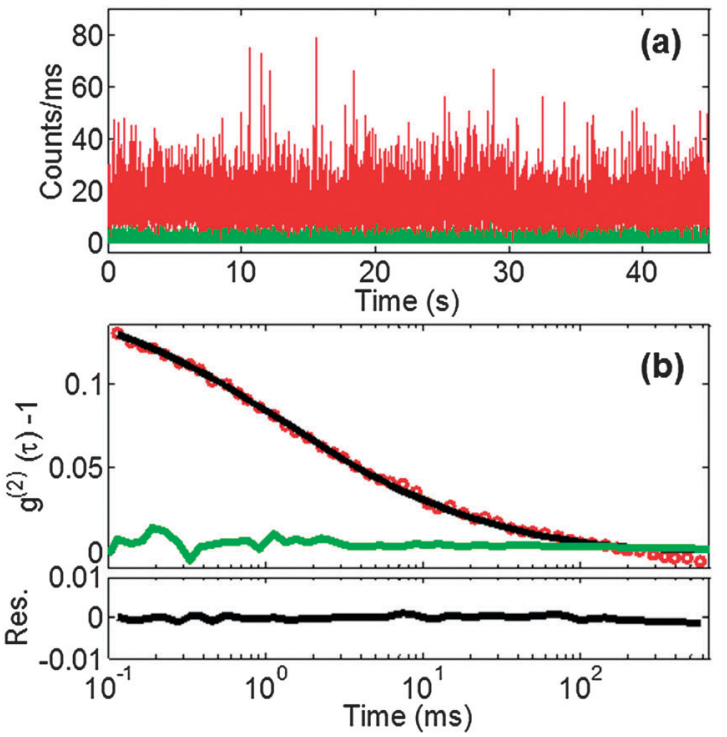

Fig. 2 (a) Typical fluorescence time trace taken on a nanorod (red) and on the background where no nanorod was present (green). The excitation power was $10 \mu \mathrm{W}$ at the sample. The data was binned to $1 \mathrm{~ms}$ for better visualization. (b) Autocorrelation curves of the time traces (colors correspond to (a)). A double exponential fit (black) to the red curve yields correlation times of $1.1 \mathrm{~ms}$ and $105 \mathrm{~ms}$. Residuals from the fit are shown in the lower panel (black line). No correlation was obtained from the fluorescence time trace recorded on the background (green). previous studies where we show single-molecule fluorescence enhancement by individual gold nanorods. We note that fluorescence time traces are recorded by time tagging individual photons to avoid binning. In Fig. 2a we used $1 \mathrm{~ms}$ bin time for visualization. The correlation analysis is performed on the raw data.

The autocorrelation of the fluorescence time traces is shown in Fig. 2b. The green curve, calculated from the time trace recorded without a nanorod does not show any visible correlation. This is expected because at the given CV concentration of $1 \mu \mathrm{M}$, more than 1000 molecules are already present in the focus volume $(\sim 1 \mathrm{fL})$. Therefore, the fluctuations of fluorescence intensity due to molecules diffusing in or out of the focal volume are too small to detect under the experimental noise. However, we see clear correlation from the time trace recorded on a gold nanorod (red circles). We have fitted the autocorrelation trace with a double exponential decay profile (black line) with a fast component of $1.1 \mathrm{~ms}$ and a slow-component of $105 \mathrm{~ms}$. Below we discuss these components in detail.

To assign the origin of these components we first calculate the diffusion time of a CV molecule through the near field of a gold nanorod. For this calculation we need: (a) the near-field region of a nanorod, with its shape and volume, and (b) the effective viscosity a CV molecule experiences while moving through the near field. The near-field map of a nanoparticle is difficult to measure experimentally and one has to rely on theory. Fig. S2 (ESI $\dagger$ ) shows the calculated near-field intensity map of a $25 \mathrm{~nm} \times 47 \mathrm{~nm}$ gold nanorod excited at its longitudinal SPR. ${ }^{27}$ The field-intensity decreases sharply away from the tip. It is divided by a factor 10 at a distance of about $10 \mathrm{~nm}$. Assuming this spatial dependence to be the steepest one, we use this dimension of the near-field area, about $10 \mathrm{~nm}$, as the one determining the burst duration for diffusing molecules.

The viscosity of glycerol depends strongly on temperature. ${ }^{31}$ Hence to calculate the local viscosity of glycerol in the nearfield, we need to know its temperature which is expected to be higher than the ambient temperature due to the laser-induced heating of the gold nanorod. The steady-state temperature distribution around a nanorod of $25 \mathrm{~nm} \times 60 \mathrm{~nm}$ is shown in Fig. S3 (ESI $\dagger$ ). The excitation power was $10 \mu \mathrm{W}$ and the absorption cross section of the nanorod is $10^{4} \mathrm{~nm}^{2}$ at the excitation wavelength of $633 \mathrm{~nm}$. The maximum temperature rise at the surface of the nanorod is estimated to be $\sim 2.4 \mathrm{~K}$. The temperature rise decreases sharply as one moves further away from the surface of the nanorod and becomes negligible at a distance of $\sim 100 \mathrm{~nm}$.

This temperature gradient will result in a viscosity gradient within the near-field and calculating the diffusion time of a molecule through such a viscosity gradient is relatively complex $^{32}$ and beyond the scope of the simple demonstration presented in this manuscript. Here we simplify the viscosity gradient with an average viscosity corresponding to a temperature which is the mean of the temperatures at nanorod's surface and at $10 \mathrm{~nm}$ away (near-field thickness) from it. Using such a simplification, we estimate the molecule's diffusion time to $0.5 \mathrm{~ms}$ (see ESI $\dagger$ ). This estimated value of the diffusion 
time agrees fairly well with our experimentally determined fast component of $1.1 \mathrm{~ms}$. This indicates that the fast component could be due to the free diffusion of $\mathrm{CV}$ molecules through the near field of the nanorods. We note that molecular diffusion should give rise to a non-exponential dependence of the correlation function. For example, diffusion in 2 dimensions produces an algebraic decay of the form $\left(1+\tau / \tau_{\mathrm{D}}\right)^{-1}$. However, the noise in our current measurements is too large to detect deviations from a single exponential decay, which would be the signature of a diffusion process.

The slow component on the other hand has a correlation time two orders of magnitude longer than the estimated diffusion time through the near-field and hence it can't be associated to free diffusion of CV molecules. We can exclude the possibility that this component is due to diffusion of dyes through the diffractionlimited laser focal volume based on the fact that no correlation was observed when the time trace was measured without a nanorod (Fig. 2). We assign this slow component to sticking of the dyes onto the substrate within a nanorod's near-field and to their subsequent bleaching or desorption. Thus the correlation time of the slow component corresponds to the average bleaching time of a CV molecule in the near-field of a nanorod. Such events of stickingbleaching onto the substrate near bowtie antennas have also been reported previously by the Moerner group. ${ }^{16}$

To further support our assignment of these components, we studied the excitation power dependence of the correlation times. The motivation of this experiment is that the components, if assigned correctly above, are expected to show different behaviors with respect to the excitation power. The fast component should depend on the viscosity of glycerol, which would depend on the local temperature. The slow component, on the other hand, is the average bleaching time and hence should decrease inversely with increasing excitation power, at least below saturation.

Autocorrelation traces measured on a gold nanorod with different excitation powers are shown in Fig. 3a. We see a gradual decrease of correlation times at higher excitation power. This observation is further confirmed in Fig. $3 \mathrm{~b}$ and $\mathrm{c}$ where we plotted the averaged correlation times (fast component in Fig. 3a and slow component in Fig. $3 \mathrm{~b}$ ) as functions of excitation laser power. The points are averages of measurements on 7 individual gold nanorods. We note that even though both components get shorter at higher excitation power, the slow component shows a much more pronounced effect. The correlation time of the slow component decreases by factor of 10 while that of the fast component only decreases by a factor of 2 under the same excitation power.

The decrease of the correlation time $\left(\tau_{1}\right)$ of the fast component with increasing laser excitation power could arise from a decrease of the effective viscosity in the near-field due to increased heating at higher excitation power. The effective viscosity, $\eta_{\text {eff }}$, as a function of excitation powers can be approximated by:

$$
\eta_{\mathrm{eff}}=\eta\left(T_{\mathrm{A}}+a I\right)
$$

where $T_{\mathrm{A}}$ is the ambient temperature ( $293 \mathrm{~K}$ ), $I$ is the excitation intensity, and $a$ is a coefficient which denotes an average temperature rise over the near-field volume per unit increase of excitation power. For pure diffusion, correlation times $\left(\tau_{1}\right)$
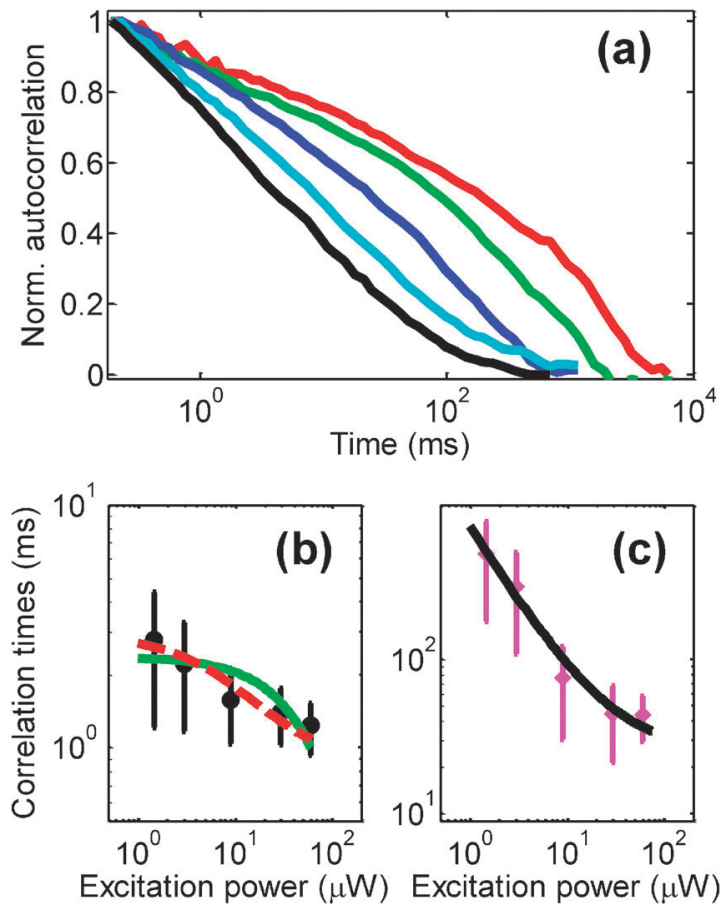

Fig. 3 (a) Autocorrelation traces measured on a gold nanorod as functions of excitation laser power (red: $1.7 \mu \mathrm{W}$, green: $3 \mu \mathrm{W}$, blue: $10 \mu \mathrm{W}$, cyan: $30 \mu \mathrm{W}$, and black: $60 \mu \mathrm{W}$ ). Excitation power dependence of the fast (b, black circles) and slow components ( $c$, magenta diamonds) calculated from bi-exponential fitting of the autocorrelation curves. The error bars are calculated from 7 individual nanorods. The green curve in (b) shows a fit to the data according to $\tau_{1} \propto \eta(T+$ al) where $T$ is the ambient temperature $(293 \mathrm{~K}), I$ is the excitation intensity and $a$ is an adjustable parameter. The red dashed line in (b) is a fit with a standard model for triplet saturation and two fit parameters. The black curve in (c) is a fit to the data using a photobleaching rate proportional to intensity, with saturation at high power.

are expected to be proportional to $\eta_{\text {eff. }}$ Indeed, the measured correlation times fits reasonably well with $\tau_{1} \propto \eta\left(T_{\mathrm{A}}+a I\right)$ as shown by the green solid curve in Fig. $3 \mathrm{~b}$. This supports an assignment of the fast component to diffusion of molecules through the near field of the nanorod. The fitting yields a

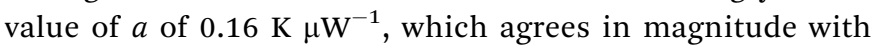

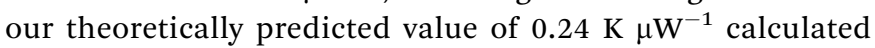
for a $10 \mathrm{~nm}$ thick near-field shell of a $25 \times 60 \mathrm{~nm}$ gold nanorod suspended in glycerol and excited at its resonance (see ESI $\dagger$ ).

An alternative or partial explanation for this fast component would be blinking from the triplet state of $\mathrm{CV}$. The triplet lifetime of a molecule typically ranges between several $\mathrm{ms}$ and hundreds of microseconds, which covers the measured correlation times of the fast component. The measured correlation time of the fast component as a function of excitation intensity is also consistent with a triplet state (red dashed line in Fig. 3b). The fit curve follows from the standard intensity dependence of triplet blinking: ${ }^{33}$

$$
\tau_{1}^{-1}=A_{1}+B_{1} \frac{I}{I+I_{\mathrm{s}}},
$$


the intersystem crossing rates $A_{1}$ and $B_{1}$ being adjustable parameters.

The triplet state of $\mathrm{CV}$, and in fact of all triphenylmethane dyes, is difficult to populate due to very efficient non-radiative decay of the excited $\mathrm{S}_{1}$ state. $^{34}$ The non-radiative decay is attributed to the dye's structural flexibility, which depends on the viscosity of the solvent. In solvents with high viscosities a low population of triplet state was reported for $\mathrm{CV} .{ }^{34}$ FCS measurement on a dilute solution of CV (10 pM) in glycerol indeed shows a component with a correlation time of $17 \mathrm{~ms}$ (Fig. S6, ESI $\dagger$ ), two orders of magnitude shorter than translational diffusion $(\sim 1 \mathrm{~s})$ and three orders of magnitude longer than rotational diffusion (a few $\mu \mathrm{s}$ ). This component could be associated to the triplet blinking of CV. Therefore, we can't exclude a contribution from triplet state blinking to the fast component. Further experiments are required to clarify this question.

The correlation time of the long component $\left(\tau_{2}\right)$, on the other hand, shows an inverse linear dependence on the excitation power (Fig. 3c) for low excitation power with a possible inflection for excitation power above $30 \mu \mathrm{W}$. The inverse linear dependence is consistent with a sticking-bleaching mechanism as the bleaching time of a molecule (i.e. correlation time of the long component) is inversely proportional to the excitation laser intensity. ${ }^{33}$ The possible saturation at high excitation intensity is due to saturation of the dye. A molecule can only absorb a certain maximum number of photons defined by its saturation limit and hence increasing excitation intensity above its saturation does not reduce its bleaching time. Indeed, the correlation times (black line in Fig. 3c) are well fitted with the expression $1 / \tau_{2} \propto\left(1+\frac{I_{\mathrm{s}}}{I}\right)$, which includes saturation of the molecule.

\section{Conclusions and outlook}

In this manuscript we have demonstrated that wet-chemically synthesized gold nanorods, thanks to the strong electromagnetic field-enhancement at their tips, enable one to perform FCS on weak fluorophores at micromolar concentration. Given the ease of synthesis and tunability of their SPR wavelengths we expect gold nanorods to be complementary to lithographic nanoantennas. Lithography allows preparing arrays of antenna on a substrate which can be handy for parallel processing. Chemically synthesized nanorods, on the other hand, will have an advantage in many complex environments where a solid substrate cannot be incorporated.

\section{Acknowledgements}

S. K., H. Y., and M. O. acknowledge financial support from the ERC Advanced Grant SiMoSoMa. S. K. acknowledges financial support from the Netherlands Organization for Scientific Research (NWO, ECHO grant). H.Y. acknowledges a postdoctoral fellowship from FWO (Research Foundation Flanders). We thank Pedro M. R. Paulo for the numerical simulations.

\section{References}

1 O. Krichevsky and G. Bonnet, Rep. Prog. Phys., 2002, 65, 251-297.

2 S. T. Hess, S. H. Huang, A. A. Heikal and W. W. Webb, Biochemistry, 2002, 41, 697-705.

3 K. Chattopadhyay, E. L. Elson and C. Frieden, Proc. Natl. Acad. Sci. U. S. A., 2005, 102, 2385-2389.

4 S. Doose, H. Neuweiler and M. Sauer, ChemPhysChem, 2009, 10, 1389-1398.

5 J. Stromqvist, A. Chmyrov, S. Johansson, A. Andersson, L. Maler and J. Widengren, Biophys. J., 2010, 99, 3821-3830.

6 F. J. Meyer-Almes and M. Auer, Biochemistry, 2000, 39, 13261-13268.

7 H. Craighead, Nature, 2006, 442, 387-393.

8 D. Punj, M. Mivelle, S. B. Moparthi, T. S. van Zanten, H. Rigneault, N. F. van Hulst, M. F. Garcia-Parajo and J. Wenger, Nat. Nanotechnol., 2013, 8, 512-516.

9 P. Holzmeister, G. P. Acuna, D. Grohmann and P. Tinnefeld, Chem. Soc. Rev., 2014, 43, 1014-1028.

10 M. J. Levene, J. Korlach, S. W. Turner, M. Foquet, H. G. Craighead and W. W. Webb, Science, 2003, 299, 682-686.

11 J. A. Schuller, E. S. Barnard, W. S. Cai, Y. C. Jun, J. S. White and M. L. Brongersma, Nat. Mater., 2010, 9, 193-204.

12 P. Zijlstra, P. M. R. Paulo and M. Orrit, Nat. Nanotechnol., 2012, 7, 379-382.

13 L. Novotny and N. van Hulst, Nat. Photonics, 2011, 5, 83-90.

14 A. Kinkhabwala, Z. F. Yu, S. H. Fan, Y. Avlasevich, K. Mullen and W. E. Moerner, Nat. Photonics, 2009, 3, 654-657.

15 H. Yuan, S. Khatua, P. Zijlstra, M. Yorulmaz and M. Orrit, Angew. Chem., Int. Ed., 2013, 52, 1217-1221.

16 A. A. Kinkhabwala, Z. F. Yu, S. H. Fan and W. E. Moerner, Chem. Phys., 2012, 406, 3-8.

17 H. Aouani, O. Mahboub, N. Bonod, E. Devaux, E. Popov, H. Rigneault, T. W. Ebbesen and J. Wenger, Nano Lett., 2011, 11, 637-644.

18 T. G. Habteyes, S. Dhuey, E. Wood, D. Gargas, S. Cabrini, P. J. Schuck, A. P. Alivisatos and S. R. Leone, ACS Nano, 2012, 6, 5702-5709.

19 P. Anger, P. Bharadwaj and L. Novotny, Phys. Rev. Lett., 2006, 96, 113002.

20 G. P. Acuna, F. M. Möller, P. Holzmeister, S. Beater, B. Lalkens and P. Tinnefeld, Science, 2012, 338, 506-510.

21 H. Cang, A. Labno, C. G. Lu, X. B. Yin, M. Liu, C. Gladden, Y. M. Liu and X. Zhang, Nature, 2011, 469, 385-388.

22 M. P. Busson, B. Rolly, B. Stout, N. Bonod and S. Bidault, Nat. Commun., 2012, 3, 6.

23 L. Vigderman, B. P. Khanal and E. R. Zubarev, Adv. Mater., 2012, 24, 4811-4841.

24 X. C. Ye, C. Zheng, J. Chen, Y. Z. Gao and C. B. Murray, Nano Lett., 2013, 13, 765-771. 
25 B. Nikoobakht and M. A. El-Sayed, Chem. Mater., 2003, 15, 1957-1962.

26 P. Zijlstra, A. L. Tchebotareva, J. W. M. Chon, M. Gu and M. Orrit, Nano Lett., 2008, 8, 3493-3497.

27 S. Khatua, P. M. R. Paulo, H. Yuan, A. Gupta, P. Zijlstra and M. Orrit, ACS Nano, 2014, 8, 4440-4449.

28 B. van den Broek, B. Ashcroft, T. H. Oosterkamp and J. van Noort, Nano Lett., 2013, 13, 980-986.

29 M. Yorulmaz, S. Khatua, P. Zijlstra, A. Gaiduk and M. Orrit, Nano Lett., 2012, 12, 4385-4391.
30 A. M. Funston, C. Novo, T. J. Davis and P. Mulvaney, Nano Lett., 2009, 9, 1651-1658.

31 R. Zondervan, F. Kulzer, G. C. G. Berkhout and M. Orrit, Proc. Natl. Acad. Sci. U. S. A., 2007, 104, 12628-12633.

32 D. Rings, R. Schachoff, M. Selmke, F. Cichos and K. Kroy, Phys. Rev. Lett., 2010, 105, 4.

33 R. Zondervan, F. Kulzer, M. A. Kol'chenko and M. Orrit, J. Phys. Chem. A, 2004, 108, 1657-1665.

34 D. F. Duxbury, Chem. Rev., 1993, 93, 381-433. 